\title{
Synthesis and biological assessment of novel acylhydrazone derivatives of 2-methyl-1,4-naphthoquinone
}

\author{
Kamal Bouhadir $\odot^{1 \dagger}$, Hala Atallah $\odot^{1,2 \dagger}$, Rana Mezher $\odot^{1,2}$, \\ Maamoun Fatfat $\odot^{3}$, Hala Gali-Muhtasib $\odot^{3}$ and Jomana Elaridi $\odot^{* 2}$ \\ ${ }^{1}$ Department of Chemistry, Faculty of Arts and Sciences, American University of Beirut, \\ Beirut, 11-0236, Lebanon \\ ${ }^{2}$ Department of Natural Sciences, School of Arts and Sciences, Lebanese American \\ University, Beirut, 1102-2801, Lebanon \\ ${ }^{3}$ Department of Biology, and Department of Anatomy, Cell Biology and Physiological \\ Sciences, American University of Beirut, Beirut, 11-0236, Lebanon
}

(Received July 28, 2017; Revised September 17, 2017; Accepted September 19, 2017)

\begin{abstract}
Naphthoquinones are medicinally important molecules with a diverse array of biological properties such as antimicrobial, antifungal, antiviral, anti-inflammatory, anti-artherosclerotic and anticarcinogenic activities. In this study, we report the simple and direct preparation of a new group of novel menadione-hydrazone conjugates by reaction of 2-methyl-1,4-naphthoquinones with several aliphatic, aromatic and nucleobase hydrazides. The menadione-hydrazone conjugates were produced in excellent yields and characterized by IR, NMR and HRMS. The menadione derivatives were tested for their anticancer effects against human colon cancer HCT116 and human breast cancer MCF-7 cell lines. Interestingly, the molecules displayed disparate activities against both cell lines; the menadione hydrazones derived from the lipophilic myristic hydrazide and stearic hydrazide exhibited the most potent activity against HCT116 cell lines with IC50 of 89 and $64 \mu \mathrm{M}$. The most effective compounds against MCF-7 cells were the lauric hydrazide and benzoic hydrazide-derived menadione hydrazones with IC50 of $56 \mu \mathrm{M}$.
\end{abstract}

Keywords: 2-methyl-1,4-naphthoquinone; menadione; acylhydrazone; pyrimidine nucleobase; purine nucleobase. (C) 2017 ACG Publications. All rights reserved.

\section{Introduction}

Naphthoquinones are molecules isolated from plants and have been utilized in the treatment of a multitude of illnesses and ailments. ${ }^{1}$ A multitude of naturally occurring naphthoquinones reportedly exhibit a diverse array of biological activities including fungicidal, ${ }^{2}$ antibacterial, ${ }^{3}$ antiviral, ${ }^{4}$ antimalarial, ${ }^{5}$ anticancer, ${ }^{1,6-12}$ in addition to effects that counter inflammation ${ }^{13}$ and artherosclerosis. ${ }^{14}$

\footnotetext{
* Corresponding author: E-mail: jomana.aridi@lau.edu.lb; Phone: +9611786456; Fax: +9611867098

${ }^{\dagger}$ The authors equally contributed to this work.
} 
The naphthoquinone (NQ) core $\mathbf{1}$ possesses redox properties and generally serves as key links in electron transport processes in the metabolic pathway and in multiple biological oxidative reactions. Vitamin $\mathrm{K}$, vitamin $\mathrm{E}$, and coenzyme Q, for example, participate in biological processes involving electron transport, blood clotting and oxidative phosphorylation. ${ }^{15}$<smiles>O=C1C=CC(=O)c2ccccc21</smiles>

1<smiles>CC1=CC(=O)C(C(C)C)=CC1=O</smiles>

2<smiles>[R]C(=O)NN=C1C=C(C(C)C)C(=O)C=C1C</smiles>

3<smiles>CC1=CC(=O)c2ccccc2C1=O</smiles>

4

Figure 1. Several quinone derivatives

Our interest in naphthoquinones was heightened by research we have conducted on a related project involving the synthesis and activity of thymoquinone derivatives. The black seed (Nigella Sativa) is the source of thymoquinone 2 (Figure 1) (TQ), an emerging natural medicinal compound with powerful antiproliferative, antioxidant, cytotoxic and antiinflammatory activities. ${ }^{16-21}$ Recently, hydrazone derivatives of TQ were synthesized by the reaction of TQ with long chain unsaturated hydrazides. The resultant hydrazones 3 also showed anti-cancer activity. ${ }^{22}$ Furthermore, work done by Schobert et al. reported that the inclusion of acylgydrazones derived from alkenyl groups of fatty acids at carbon- 4 of the quinone ring greatly enhanced the anticancer activity of TQ against resistant cancer cell lines.

In general, hydrazones represent an interesting class of organic compounds as substrates for pyrazoles, triazoles, triazines, oxadiazoles and other heterocyclic compounds and as biologically important molecules themselves. Hydrazones have been reported to possess anti-inflammatory, analgesic, anticonvulsant, antituberculous, antitumor, anti-HIV and antimicrobial activity. ${ }^{23-28}$

We aimed to prepare a series of NQ hydrazones derived from menadione (2-methyl1,4-naphthoquinone) 4 (Figure 1), based on the reaction of menadione with several saturated and unsaturated hydrazides. Menadione acts as the central moiety of Vitamin $\mathrm{K}$ and is essential for several biological processes including blood coagulation, bone metabolism and cell growth. ${ }^{15}$ The methyl group in the carbon-2 position of the quinone ring is reportedly essential for in vitro and in vivo activity ${ }^{29,30}$ and may enhance the lipophilicity and cell permeability of this and related compounds. In this research, we postulated that the synthesis of related hydrazones could also increase the biological activity of the parent naphthoquinone molecule. We chose various short chain and long chain aliphatic hydrazides in addition to aromatic and purine and pyrimidine-derived hydrazides. Many pyrimidine and purine nucleobases such as clofarabine, vidaza, nelarabine, fludrabine, cladribine and gemcitabine are employed for the clinical treatment of hematological cancers and solid tumors. ${ }^{31-33}$ Although there have been significant advances in the design and development of anticancer therapeutics, the synthesis of new nucleoside-based drugs for treating tumors continues to be a cardinal and far-reaching research objective. 


\section{Experimental}

\subsection{Materials and Apparatus}

Melting points were determined on a DigiMelt apparatus and were uncorrected. NMR spectra were determined in deuterated solvents with TMS as the internal standard on a Bruker AM 500 NMR spectrometer. Chemical shifts are reported in ppm $(\delta)$ downfield relative to TMS. Coupling constants were reported in hertz (Hz). Infrared spectra were recorded as $\mathrm{KBr}$ pellets using a Nicolet AVATAR 360 FTIR ESP spectrometer with a Hewlett Packard Desk jet 840C plotter and Thermo Scientific iD5 Diamond ATR for Nicolet iS5 FT-IR Spectrometer. The IR bands are reported as wave numbers $\left(\mathrm{cm}^{-1}\right)$. High-resolution mass spectra (HRMS) were recorded on a Waters SYNAPT G2-Si high definition mass spectrometer in positive electrospray ionization $(\mathrm{ESI}+)$ mode. Thin layer chromatography (TLC) was performed on polygram Sil G/UV254 silica gel sheets (used directly as received). Column chromatography employed ALDRICH silica gel (60^, 230-400 mesh). Reagents used for synthesis were purchased from the Aldrich Chemical Company (Milwaukee, WI) and ACROS Chemicals. ${ }^{1} \mathrm{H}$ and ${ }^{13} \mathrm{C}$ NMR, and HRMS spectra of compounds are provided in the Supporting Information.

\subsection{General Procedure for the Synthesis of $\mathbf{6} \boldsymbol{a}-\mathbf{m}$ :}

A solution of alkyl or aromatic hydrazide (5-100 mmol), 2-methyl-[1,4]naphthoquinone (1 equiv, 5-100 mmol) and a few drops of TFA was placed in a sealed tube in ethanol $\left(10-50 \mathrm{ml}\right.$ ) and stirred for 24 (or 72 ) hours at $90^{\circ} \mathrm{C}$. The reaction mixture was subsequently cooled to room temperature and the precipitate was filtered and washed with cold ethanol and dried in a vacuum oven at $50^{\circ} \mathrm{C}$ to afford the desired hydrazone as a solid.

$N^{\prime}$-(3-methyl-4-oxonaphthalen-1(4H)-ylidene)pentanehydrazide (6a): Yellow solid, 76\% yield, m.p: $243 \sim 245^{\circ} \mathrm{C} .{ }^{1} \mathrm{H}$ NMR $\left(500 \mathrm{MHz}, \mathrm{CDCl}_{3}\right): \delta 0.98-1.01\left(\mathrm{t}, 3 \mathrm{H}, \mathrm{CH}_{3}\right) ; 1.48-1.5(\mathrm{~m}$, $\left.2 \mathrm{H}, \mathrm{CH}_{2}\right) ; 1.62\left(\mathrm{~s}, 3 \mathrm{H}, \mathrm{CH}_{3}\right) ; 1.78-1.8\left(\mathrm{~m}, 2 \mathrm{H}, \mathrm{CH}_{2}\right) ; 2.94-2.97\left(\mathrm{t}, J=7.5 \mathrm{~Hz}, 2 \mathrm{H}, \mathrm{CH}_{2}\right) ; 7.54-$ $8.28\left(\mathrm{~m}, 5 \mathrm{H}, \mathrm{H}_{\mathrm{Ar}}\right) ; 10.94(\mathrm{~s}, 1 \mathrm{H}, \mathrm{NH}) .{ }^{13} \mathrm{C}$ NMR $\left(500 \mathrm{MHz}, \mathrm{DMSO}-d_{6}\right): \delta 13.9(\mathrm{CH} 3-4)$; $17.18\left(\mathrm{CH}_{3}-\mathrm{b}\right) ; 22.51,26.5,30.9,32.4\left(\left[\mathrm{CH}_{2}\right]_{3} 1-2-3\right) ; 122.2,123.1,126.3,129.4,132.4$ $\left(5 \mathrm{CH}_{\mathrm{Ar}} \mathrm{a}-\mathrm{c}-\mathrm{d}-\mathrm{e}-\mathrm{f}\right) ; 134.8\left(\mathrm{C}_{\mathrm{Ar}}\right) ; 137.4(\mathrm{C}=\mathrm{N}) ; 141.9\left(\mathrm{C}_{\mathrm{Ar}}\right) ; 177.9(\mathrm{C}=\mathrm{O}$ amide $) ; 185\left(\mathrm{C}_{=} \mathrm{O}_{\text {ketone }}\right)$; $207.0\left(\mathrm{C}_{\mathrm{Ar}}\right)$ FTIR $\left(\mathrm{cm}^{-1}\right): 700 \mathrm{~m}, 770 \mathrm{~s}, 1171 \mathrm{~m}, 1287 \mathrm{~m}, 1398 \mathrm{~m}, 1637 \mathrm{~s}, 1668 \mathrm{~s}, 3050 \mathrm{w}$. HRMS (ESI ${ }^{+}, \mathrm{MeOH}$ ): Found: $m / z 271.1447\left[(\mathrm{M}+\mathrm{H})^{+}\right], \mathrm{C}_{16} \mathrm{H}_{19} \mathrm{~N}_{2} \mathrm{O}_{2}$ requires 271.1445.

$N^{\prime}$-(3-Methyl-4-oxonaphthalen-1(4H)-ylidene)octanehydrazide (6b): Yellow solid, 65\% yield, m.p. $209-212^{\circ} \mathrm{C} .{ }^{1} \mathrm{H}$ NMR $\left(500 \mathrm{MHz}, \mathrm{CDCl}_{3}\right): \delta 0.89(\mathrm{t}, J=6.9 \mathrm{~Hz}, 3 \mathrm{H}), 1.30-1.83(\mathrm{~m}, 10 \mathrm{H})$, $2.27(\mathrm{~s}, 3 \mathrm{H}), 2.94(\mathrm{t}, J=7.5 \mathrm{~Hz}, 2 \mathrm{H}), 7.55(\mathrm{t}, J=7.5 \mathrm{~Hz}, 1 \mathrm{H}), 7.64(\mathrm{t}, J=7.6 \mathrm{~Hz}, 1 \mathrm{H}), 7.88$ $(\mathrm{s}, 1 \mathrm{H}), 8.18(\mathrm{~d}, J=7.9 \mathrm{~Hz}, 1 \mathrm{H}), 8.27(\mathrm{~d}, J=8.0 \mathrm{~Hz}, 1 \mathrm{H}), 11.06(\mathrm{~s}, 1 \mathrm{H}) ;{ }^{13} \mathrm{C}$ NMR $(125 \mathrm{MHz}$, $\mathrm{CDCl}_{3}$-d6): $\delta 14.0 ; 17.0,22.6,24.4,24.7,31.6,32.7,32.9,122.4,122.5,123.1,126.4,129.4$, 132.4, 134.9, 137.6, 141.0, 178.0, 185.1; FTIR $\left(\mathrm{cm}^{-1}\right): 2849,2926,1670,1641,1560,1542$, 1466, 1399, 1376, 1332, 1288, 1242, 1203, 1171, 1078, 1017, 952, 889, 867, 773. Mass spectrum (ESI-, C18 column, $50 \% \mathrm{MeOH} / 50 \% \mathrm{DI}$ ): $\mathrm{m} / \mathrm{z}$ calcd for $\mathrm{C}_{19} \mathrm{H}_{23} \mathrm{~N}_{2} \mathrm{O}_{2}\left[(\mathrm{M}-\mathrm{H})^{-}\right]$ 311.2, found 311.0. HRMS (ESI $\left.{ }^{+}, \mathrm{MeOH}\right)$ : Found: $m / z 313.1916\left[(\mathrm{M}+\mathrm{H})^{+}\right], \mathrm{C}_{19} \mathrm{H}_{25} \mathrm{~N}_{2} \mathrm{O}_{2}$ requires 313.1915 .

$N^{\prime}$-(3-Methyl-4-oxonaphthalen-1(4H)-ylidene)undec-10-enehydrazide (6c): Yellow solid, $67 \%$ yield, m.p. $187-190^{\circ} \mathrm{C} .{ }^{1} \mathrm{H}$ NMR $\left(500 \mathrm{MHz}, \mathrm{CDCl}_{3}\right): \delta 1.31-1.82(\mathrm{~m}, 14 \mathrm{H}), 2.26(\mathrm{~s}, 3 \mathrm{H})$, $2.95(\mathrm{t}, J=7.5 \mathrm{~Hz}, 2 \mathrm{H}),, 4.96(\mathrm{~m}, 2 \mathrm{H}), 5.80(\mathrm{~m}, 1 \mathrm{H}), 7.55(\mathrm{t}, J=7.5 \mathrm{~Hz}, 1 \mathrm{H}), 7.64(\mathrm{t}, J=$ $8.3 \mathrm{~Hz}, 1 \mathrm{H}), 7.88(\mathrm{~s}, 1 \mathrm{H}), 8.18(\mathrm{~d}, J=7.9 \mathrm{~Hz}, 1 \mathrm{H}), 8.26(\mathrm{~d}, J=8.0 \mathrm{~Hz}, 1 \mathrm{H}), 11.08(\mathrm{~s}, 1 \mathrm{H}) ;{ }^{13} \mathrm{C}$ 
NMR (125 MHz, $\left.\mathrm{CDCl}_{3}\right): \delta 14.1,17.1,22.6,24.5,24.9,31.8,31.9,32.8,33.8,114.3,123.0$ 123.2, 126.5, 129.3, 130.7, 132.5, 134.7, 137.8, 139.2, 141.0, 176.5, 185.3; FTIR $\left(\mathrm{cm}^{-1}\right)$ : 1679, 1643, 1601, 1546, 1442, 1391, 1288, 1242, 1078, 907, 892, 769, 740, 696. Mass spectrum (ESI-, C18 column, $50 \% \mathrm{MeOH} / 50 \%$ DI): $\mathrm{m} / z$ calcd for $\mathrm{C}_{22} \mathrm{H}_{27} \mathrm{~N}_{2} \mathrm{O}_{2} \quad\left[(\mathrm{M}-\mathrm{H})^{-}\right]$ 351.2, found 351.0. HRMS (ESI ${ }^{+}, \mathrm{MeOH}$ ): Found: $m / z 353.2229\left[(\mathrm{M}+\mathrm{H})^{+}\right], \mathrm{C}_{22} \mathrm{H}_{29} \mathrm{~N}_{2} \mathrm{O}_{2}$ requires 353.2230 .

$N^{\prime}$-(3-Methyl-4-oxonaphthalen-1(4H)-ylidene)dodecanehydrazide (6d): Yellow solid, 64\% yield, m.p. $189-191^{\circ} \mathrm{C} .{ }^{1} \mathrm{H}$ NMR $\left(500 \mathrm{MHz}, \mathrm{CDCl}_{3}\right): \delta 0.87(\mathrm{t}, J=6.9 \mathrm{~Hz}, 3 \mathrm{H}), 1.27-1.81$ $(\mathrm{m}, 18 \mathrm{H}), 2.27(\mathrm{~s}, 3 \mathrm{H}), 2.95(\mathrm{t}, J=7.5 \mathrm{~Hz}, 2 \mathrm{H}), 7.55(\mathrm{t}, J=7.5 \mathrm{~Hz}, 1 \mathrm{H}), 7.64(\mathrm{t}, J=7.6 \mathrm{~Hz}$, $1 \mathrm{H}), 7.89(\mathrm{~s}, 1 \mathrm{H}), 8.18(\mathrm{~d}, J=7.9 \mathrm{~Hz}, 1 \mathrm{H}), 8.26(\mathrm{~d}, J=7.6 \mathrm{~Hz}, 1 \mathrm{H}), 11.12(\mathrm{~s}, 1 \mathrm{H}) ;{ }^{13} \mathrm{C} \mathrm{NMR}$ $\left(125 \mathrm{MHz}, \mathrm{CDCl}_{3}\right.$ ): $\delta 14.1,17.1,22.7,24.5,29.4$ (x2), 29.5, $29.6(\mathrm{x} 2), 29.7,31.9,32.8,122.8$, 123.1, 126.4, 129.4, 132.3, 134.9, 137.7, 130.7, 140.9, 178.4, 185.1; FTIR $\left(\mathrm{cm}^{-1}\right): 2988,2918$, 2851, 1682, 1643, 1387, 1252, 1235, 1171, 1078, 1066, 1057, 892, 767, 695. Mass spectrum (ESI-, C18 column, 50\% MeOH / 50\% DI): $\mathrm{m} / z$ calcd for $\mathrm{C}_{23} \mathrm{H}_{31} \mathrm{~N}_{2} \mathrm{O}_{2}\left[(\mathrm{M}-\mathrm{H})^{-}\right] 367.2$, found 367.3. HRMS (ESI ${ }^{+}, \mathrm{MeOH}$ ): Found: $m / z 369.2542\left[(\mathrm{M}+\mathrm{H})^{+}\right], \mathrm{C}_{23} \mathrm{H}_{33} \mathrm{~N}_{2} \mathrm{O}_{2}$ requires 369.2540.

$N^{\prime}$-(3-Methyl-4-oxonaphthalen-1(4H)-ylidene)tetradecanehydrazide (6e): Yellow solid, $60 \%$ yield, m.p. $180-182^{\circ} \mathrm{C} .{ }^{1} \mathrm{H}$ NMR $\left(500 \mathrm{MHz}, \mathrm{CDCl}_{3}\right): \delta 0.87(\mathrm{t}, J=6.8 \mathrm{~Hz}, 3 \mathrm{H}), 1.25-1.83(\mathrm{~m}$, 22H), $2.27(\mathrm{~s}, 3 \mathrm{H}), 2.95(\mathrm{t}, J=7.5 \mathrm{~Hz}, 2 \mathrm{H}), 7.55(\mathrm{t}, J=7.5 \mathrm{~Hz}, 1 \mathrm{H}), 7.64(\mathrm{t}, J=7.6 \mathrm{~Hz}, 1 \mathrm{H})$, $7.89(\mathrm{~s}, 1 \mathrm{H}), 8.18(\mathrm{~d}, J=7.9 \mathrm{~Hz}, 1 \mathrm{H}), 8.26(\mathrm{~d}, J=7.9 \mathrm{~Hz}, 1 \mathrm{H}), 11.11(\mathrm{~s}, 1 \mathrm{H}) ;{ }^{13} \mathrm{C}$ NMR $(125$ $\left.\mathrm{MHz}, \mathrm{CDCl}_{3}\right): \delta 14.1,17.1,22.7,24.5,29.4(\mathrm{x} 2), 29.5,29.6,29.7(\mathrm{x} 3), 30.9,31.9,32.8,122.7$, 123.1, 126.4, 129.4, 132.4, 134.9, 137.6, 130.7, 140.9, 178.2, 185.1; FTIR $\left(\mathrm{cm}^{-1}\right): 2917,2850$, 1682, 1643, 1602, 1548, 1442, 1388, 1286, 1260, 1245, 1225, 1171, 1155, 1115, 1078, 1031, 952, 892, 767. HRMS (ESI $\left.{ }^{+}, \mathrm{MeOH}\right)$ : Found: $m / z$ 397.2855 [(M+H) $\left.{ }^{+}\right], \mathrm{C}_{25} \mathrm{H}_{37} \mathrm{~N}_{2} \mathrm{O}_{2}$ requires 397.2852 .

$N^{\prime}$-(3-Methyl-4-oxonaphthalen-1(4H)-ylidene)stearohydrazide (6f): Yellow solid, 79\% yield, m.p. $166-169^{\circ} \mathrm{C} .{ }^{1} \mathrm{H}$ NMR $\left(500 \mathrm{MHz}, \mathrm{CDCl}_{3}\right): \delta 0.88(\mathrm{t}, J=6.8 \mathrm{~Hz}, 3 \mathrm{H}), 1.25-1.81(\mathrm{~m}, 30 \mathrm{H})$, $2.26(\mathrm{~s}, 3 \mathrm{H}), 2.95(\mathrm{t}, J=7.5 \mathrm{~Hz}, 2 \mathrm{H}), 7.54(\mathrm{t}, J=7.8 \mathrm{~Hz}, 1 \mathrm{H}), 7.65(\mathrm{t}, J=7.3 \mathrm{~Hz}, 1 \mathrm{H}), 7.82$ $(\mathrm{s}, 1 \mathrm{H}), 8.18(\mathrm{~d}, J=7.8 \mathrm{~Hz}, 1 \mathrm{H}), 8.27(\mathrm{~d}, J=7.9 \mathrm{~Hz}, 1 \mathrm{H}), 10.88(\mathrm{~s}, 1 \mathrm{H}) ;{ }^{13} \mathrm{C}$ NMR $(125 \mathrm{MHz}$, $\left.\mathrm{CDCl}_{3}\right): \delta 14.1,17.2,22.6,22.7(\mathrm{x} 2), 24.4,24.5(\mathrm{x} 2), 29.4(\mathrm{x} 2), 29.5(\mathrm{x} 2), 29.7(\mathrm{x} 2), 31.9$ (x2), 32.8 (x2), 121.8, 123.1, 126.4, 129.5, 132.5, 137.3, 134.8, 130.6, 141.1, 177.5, 185.1; FTIR $\left(\mathrm{cm}^{-1}\right): 2917,2849,1681,1644,1602,1548,1467,1289,1259,1245,1229,1171,1076$, 767, 719, 695. Mass spectrum (ESI+, C18 column, 50\% MeOH / 50\% DI): $\mathrm{m} / z$ calcd for $\mathrm{C}_{29} \mathrm{H}_{44} \mathrm{~N}_{2} \mathrm{O}_{2}\left[(\mathrm{M}+\mathrm{Na})^{+}\right]$475.3, found 475.3. HRMS (ESI $\left.{ }^{+}, \mathrm{MeOH}\right)$ : Found: $\mathrm{m} / z 453.3481$ $\left[(\mathrm{M}+\mathrm{H})^{+}\right], \mathrm{C}_{29} \mathrm{H}_{45} \mathrm{~N}_{2} \mathrm{O}_{2}$ requires 453.3480. $\mathrm{C}_{29} \mathrm{H}_{44} \mathrm{~N}_{2} \mathrm{O}_{2}$ calcd $\mathrm{C}, 76.95 ; \mathrm{H}, 9.80 ; \mathrm{N}, 6.19 \%$; found $\mathrm{C}, 76.728 ; \mathrm{H}, 9.776 ; \mathrm{N}, 6.200 \%$.

$N^{\prime}$-(3-Methyl-4-oxonaphthalen-1(4H)-ylidene)benzohydrazide (6g): Yellow solid, 63\% yield, m.p. $244-247^{\circ} \mathrm{C} .{ }^{1} \mathrm{H}$ NMR (500 MHz, DMSO-d $\left.{ }_{6}\right): \delta 2.13(\mathrm{~s}, 3 \mathrm{H}), 7.56-8.24(\mathrm{~m}, 9 \mathrm{H}), 8.24(\mathrm{~s}$, $1 \mathrm{H},), 12.02(\mathrm{~s}, 1 \mathrm{H}) ;{ }^{13} \mathrm{C}$ NMR $\left(125 \mathrm{MHz}, \mathrm{DMSO}-\mathrm{d}_{6}\right): \delta 17.0,123.9,125.2,125.8,126.1$, 128.7, 129.2, 130.0, 130.5, 132.5, 133.1, 133.9, 135.2, 135.4, 139.3, 140.7, 165.5, 184.9; FTIR $\left(\mathrm{cm}^{-1}\right): 2988,2901,1652,1638,1598,1445,1331,1312,1286,1169,1066,950,891$, 769, 708, 698, 657. Mass spectrum (ESI-, C18 column, 50\% MeOH / 50\% DI): $\mathrm{m} / z$ calcd for $\mathrm{C}_{18} \mathrm{H}_{13} \mathrm{~N}_{2} \mathrm{O}_{2}$ [(M-H)'] 289.1, found 288.9. HRMS (ESI $\left.{ }^{+}, \mathrm{MeOH}\right)$ : Found: $m / z 291.1134$ $\left[(\mathrm{M}+\mathrm{H})^{+}\right], \mathrm{C}_{18} \mathrm{H}_{15} \mathrm{~N}_{2} \mathrm{O}_{2}$ requires 291.1132. $\mathrm{C}_{18} \mathrm{H}_{14} \mathrm{~N}_{2} \mathrm{O}_{2}$ calcd $\mathrm{C}, 74.47 ; \mathrm{H}, 4.86 ; \mathrm{N}, 9.65 \%$; found $\mathrm{C}, 74.343 ; \mathrm{H}, 4.878 ; \mathrm{N}, 9.684 \%$.

2-(2,4-dioxo-3,4-dihydropyrimidin-1(2H)-yl)-N'-(3-methyl-4-oxonaphthalen-1(4H)-

ylidene) acetohydrazide (6h): Yellow solid, 91\% yield, m.p. $>260^{\circ} \mathrm{C} .{ }^{1} \mathrm{H}$ NMR $(500 \mathrm{MHz}$, DMSO- $\left.d_{6}\right): \delta 2.10\left(\mathrm{~s}, 3 \mathrm{H}, \mathrm{CH}_{3}-\mathrm{b}\right) ; 4.7,5.12\left(\mathrm{~s}, 2 \mathrm{H}, \mathrm{CH}_{2}\right) ; 5.60-5.65(\mathrm{~d}, J=6.5 \mathrm{~Hz}, 1 \mathrm{H}-1$ ); 7.64-8.33 (m, 6H, $\left.\mathrm{H}_{\mathrm{Ar}}\right) ; 11.40(\mathrm{~s}, 1 \mathrm{H}, \mathrm{NH}-5), 12.26(\mathrm{~s}, 1 \mathrm{H}, \mathrm{NH}-3) .{ }^{13} \mathrm{C}$ NMR (500 MHz, 
DMSO- $\left.d_{6}\right): \delta 17.1\left(\mathrm{CH}_{3}-\mathrm{b}\right), 49.6\left(\mathrm{CH}_{2}-4\right), 101.3,123.8,124.4,126.1,130.1\left(\left[\mathrm{CH}_{\mathrm{Ar}}\right]_{5}\right), 130.4$ $\left(\mathrm{C}_{\mathrm{Ar}}\right) ; 133.2\left(\mathrm{CH}_{\mathrm{Ar}}\right) ; 134.6,137.5,140.0\left(\left[\left(\mathrm{C}_{\mathrm{Ar}}\right)_{3}\right] ; 146.8\left(\mathrm{CH}_{\mathrm{Ar}}\right), 151.6,164.3,170.7,184.7\right.$ $\left([\mathrm{C}=\mathrm{O}]_{4}\right)$. FTIR (neat): $534 \mathrm{w}, 1169 \mathrm{w}, 1268 \mathrm{~m}, 1395 \mathrm{~m}, 1409 \mathrm{~m}, 1598 \mathrm{~m}, 1638 \mathrm{~s}, 1685 \mathrm{~s}, 3191 \mathrm{~m}$, 3445s. HRMS (ESI $\left.{ }^{+}, \mathrm{MeOH}\right):$ Found: $m / z 339.1093\left[(\mathrm{M}+\mathrm{H})^{+}\right], \mathrm{C}_{18} \mathrm{H}_{15} \mathrm{~N}_{2} \mathrm{O}_{2}$ requires 339.1094. LCMS (ESI ${ }^{+}, \mathrm{C} 18$ column, $75 \% \mathrm{MeOH} / 25 \% \mathrm{H} 2 \mathrm{O}$ ): $\mathrm{m} / z\left[\mathrm{M}-\mathrm{H}^{+}\right]$calcd for $\mathrm{C}_{17} \mathrm{H}_{13} \mathrm{~N}_{4} \mathrm{O}_{4}$ 337.1, found 337.0. HRMS (ESI $\left.{ }^{+}, \mathrm{MeOH}\right)$ : Found: $m / z 339.1094\left[(\mathrm{M}+\mathrm{H})^{+}\right]$, $\mathrm{C}_{17} \mathrm{H}_{15} \mathrm{~N}_{4} \mathrm{O}_{4}$ requires 339.1093 .

3-(2,4-dioxo-3,4-dihydropyrimidin-1(2H)-yl)-N'-(3-methyl-4-oxonaphthalen-1(4H)-

ylidene)propanehydrazide (6i): Yellow solid, 73\% yield, m.p. $>260^{\circ} \mathrm{C} .{ }^{1} \mathrm{H}$ NMR $(500 \mathrm{MHz}$, DMSO-d $\left.d_{6}\right): \delta 2.09$ (s, 3H, $\left.\mathrm{CH}_{3}-\mathrm{b}\right) ; 3.32\left(\mathrm{~m}, 2 \mathrm{H}, \mathrm{CH}_{2}\right) ; 4.02\left(\mathrm{~m}, 2 \mathrm{H}, \mathrm{CH}_{2}-4\right) ; 5.53(\mathrm{~d}, J=8.0$ $\mathrm{Hz}, 1 \mathrm{H}-2) ; 7.61-8.27\left(\mathrm{~m}, 6 \mathrm{H}, \mathrm{H}_{\mathrm{Ar}}\right) .{ }^{13} \mathrm{C}$ NMR $\left(500 \mathrm{MHz}, \mathrm{DMSO}-d_{6}\right): \delta 16.6\left(\mathrm{CH}_{3}-\mathrm{b}\right) ; 31.6$ $\left(\mathrm{CH}_{2}-5\right) ; 44.0\left(\mathrm{CH}_{2}-4\right) ; 100.1,100.5\left(\mathrm{CH}_{\mathrm{Ar}}[1-2]\right) ; 123.1\left(\mathrm{CH}_{\mathrm{Ar}}\right) ; 124.0\left(\mathrm{CH}_{\mathrm{Ar}}-\mathrm{a}\right) ; 125.5$, 129.3, $129.8\left(\left[\mathrm{CH}_{\mathrm{Ar}}\right]_{3}\right] ; 132.6\left(\mathrm{CH}_{\mathrm{Ar}}-1\right) ; 138.8\left(\mathrm{C}_{\mathrm{Ar}}\right) ; 142.1\left(\mathrm{C}_{\mathrm{Ar}}\right) ; 146.3\left(\mathrm{CH}_{\mathrm{Ar}}\right) ; 150.8,151.4$, 163.7, 164.3, $184.2\left([\mathrm{C}=\mathrm{O}]_{4}\right) . \mathrm{FTIR}\left(\mathrm{cm}^{-1}\right): 1167 \mathrm{w}, 1267 \mathrm{~m}, 1388 \mathrm{w}, 1422 \mathrm{w}, 1551 \mathrm{w}, 1644 \mathrm{~m}$, 1686s, 3028m. HRMS (ESI $\left.{ }^{+}, \mathrm{MeOH}\right)$ : Found: $m / z 353.1250\left[(\mathrm{M}+\mathrm{H})^{+}\right], \mathrm{C}_{18} \mathrm{H}_{17} \mathrm{~N}_{4} \mathrm{O}_{4}$ requires 353.1245. LCMS (ESI, C18 column, $\mathrm{MeOH} 75 \% / \mathrm{H}_{2} \mathrm{O} 25 \%$ ): $\mathrm{m} / z$ calcd for $\mathrm{C}_{18} \mathrm{H}_{15} \mathrm{~N}_{4} \mathrm{O}_{4}$ [(M-H)'] 351.1 found 350.9. HRMS $\left(\mathrm{ESI}^{+}, \mathrm{MeOH}\right)$ : Found: $m / z 353.1249\left[(\mathrm{M}+\mathrm{H})^{+}\right]$, $\mathrm{C}_{18} \mathrm{H}_{17} \mathrm{~N}_{4} \mathrm{O}_{4}$ requires 353.1250 .

2-(5-methyl-2,4-dioxo-3,4-dihydropyrimidin-1(2H)-yl)-N'-(3-methyl-4-oxonaphthalen-1(4H)ylidene)acetohydrazide (6j): Yellow solid, $85 \%$ yield, m.p. $>260^{\circ} \mathrm{C} .{ }^{1} \mathrm{H}$ NMR $(500 \mathrm{MHz}$, DMSO-d $\left.d_{6}\right): \delta 1.79\left(\mathrm{~s}, 3 \mathrm{H}, \mathrm{CH}_{3}-1\right) ; 2.11\left(\mathrm{~s}, 3 \mathrm{H}, \mathrm{CH}_{3}-\mathrm{b}\right) ; 5.05\left(\mathrm{~s}, 2 \mathrm{H}, \mathrm{CH}_{2}-4\right) ; 7.55-8.32(\mathrm{~m}$, $\left.6 \mathrm{H}, \mathrm{H}_{\mathrm{Ar}}\right) ; 11.43(\mathrm{~s}, 1 \mathrm{H}, \mathrm{NH}-5) ; 12.21(\mathrm{~s}, 1 \mathrm{H}, \mathrm{NH}-3)$. DEPT (500 MHz, DMSO-d 6 ): $\delta 12.3$ $\left(\mathrm{CH}_{3}-1\right) ; 17.1\left(\mathrm{CH}_{3}-\mathrm{b}\right) ; 49.4\left(\mathrm{CH}_{2}-4\right) ; 123.8,124.3,126.1,130.2,133.3,142.7\left(\left[\mathrm{CH}_{\mathrm{Ar}}\right]_{6}\right)$. FTIR: 550w, 775w, 1280m, 1410m, 1595m, 1595m, 1680s, 3200m. HRMS (ESI $\left.{ }^{+}, \mathrm{MeOH}\right)$ : Found: $m / z 353.1245\left[(\mathrm{M}+\mathrm{H})^{+}\right], \mathrm{C}_{18} \mathrm{H}_{17} \mathrm{~N}_{4} \mathrm{O}_{4}$ requires 353.1250. $\mathrm{C}_{18} \mathrm{H}_{16} \mathrm{~N}_{4} \mathrm{O}_{4}$ calcd: $\mathrm{C}, 61.36$; $\mathrm{H}, 4.58 ; \mathrm{N}, 15.90 \%$; found $\mathrm{C}, 61.24 ; \mathrm{H}, 4.63 ; \mathrm{N}, 15.70 \%$.

3-(5-methyl-2,4-dioxo-3,4-dihydropyrimidin-1(2H)-yl)-N'-(3-methyl-4-oxonaphthalen-1(4H)ylidene)propanehydrazide (6k): Yellow solid, $82 \%$ yield, m.p. $252-255^{\circ} \mathrm{C} .{ }^{1} \mathrm{H}$ NMR $(500$ MHz, DMSO-d6): $\delta 1.71$ (s, 3H, CH $)_{3} ; 2.09\left(\mathrm{~s}, 3 \mathrm{H}, \mathrm{CH}_{3}\right) ; 3.99\left(\mathrm{~m}, 2 \mathrm{H}, \mathrm{CH}_{2}\right) ; 3.59(\mathrm{~m}, 2 \mathrm{H}$, $\left.\mathrm{CH}_{2}\right)$ 7.55-8.26 (m, 6H, $\left.\mathrm{H}_{\mathrm{Ar}}\right) ; 11.22(\mathrm{~s}, 1 \mathrm{H}, \mathrm{NH}-6) ; 11.90(\mathrm{~s}, 1 \mathrm{H}, \mathrm{NH}-3) .{ }^{13} \mathrm{C}$ NMR $(500 \mathrm{MHz}$, DMSO-d6): $\delta 12.0\left(\mathrm{CH}_{3}-1\right) ; 16.6\left(\mathrm{CH}_{3}-\mathrm{b}\right) ; 31.8\left(\mathrm{CH}_{2}-5\right) ; 43.9\left(\mathrm{CH}_{2}-4\right) ; 108.0\left(\mathrm{C}_{\mathrm{Ar}}\right) ; 122.2$, $124.4\left(\left[\mathrm{CH}_{\mathrm{Ar}}\right]_{2}\right) ; 125.3\left(\mathrm{CH}_{\mathrm{Ar}}-\mathrm{a}\right) ; 129.3\left(\mathrm{CH}_{\mathrm{Ar}}\right) ; 129.8\left(\mathrm{C}_{\mathrm{Ar}}\right) ; 132.6\left(\mathrm{CH}_{\mathrm{Ar}}\right) ; 134.4\left(\mathrm{C}_{\mathrm{Ar}}\right) ; 136.1$ $(\mathrm{C}=\mathrm{N}) ; 138.9\left(\mathrm{C}_{\mathrm{Ar}}\right) ; 141.3\left(\mathrm{CH}_{\mathrm{Ar}}\right) ; 150.4\left(\mathrm{C}=\mathrm{O}_{\text {ketone }}\right) ; 164.2\left(\mathrm{C}=\mathrm{O}_{\text {ketone }}\right) ; 173.9\left(\mathrm{C}=\mathrm{O}_{\text {amide }}\right)$; $184.2\left([\mathrm{C}=\mathrm{O}]_{\text {ketone }}\right)$. FTIR $\left(\mathrm{cm}^{-1}\right) 695 \mathrm{~m}, 759 \mathrm{~m}, 912 \mathrm{~m}, 1163 \mathrm{~m}, 1212 \mathrm{~m}, 1283 \mathrm{~m}, 1363 \mathrm{~m}, 1478 \mathrm{w}$, $1683 \mathrm{~s}, 3000 \mathrm{w}, 3700 \mathrm{w} \mathrm{cm}{ }^{-1}$. HRMS $\left(\mathrm{ESI}^{+}, \mathrm{MeOH}\right)$ : Found: $\mathrm{m} / z$ 367.1406 [(M+H) $\left.{ }^{+}\right]$, $\mathrm{C}_{19} \mathrm{H}_{19} \mathrm{~N}_{4} \mathrm{O}_{4}$ requires 367.1407. LCMS (ESI ${ }^{+}, \mathrm{C} 18$ column, 75\% MeOH / 25\% $\mathrm{H}_{2} \mathrm{O}$ ): $\mathrm{m} / \mathrm{z}$ calcd for $\mathrm{C}_{19} \mathrm{H}_{17} \mathrm{~N}_{4} \mathrm{O}_{4}\left[(\mathrm{M}-\mathrm{H})^{-}\right]$365.1, found 364.9. HRMS (ESI ${ }^{+}, \mathrm{MeOH}$ ): Found: $m / z$ $367.1407\left[(\mathrm{M}+\mathrm{H})^{+}\right], \mathrm{C}_{19} \mathrm{H}_{19} \mathrm{~N}_{4} \mathrm{O}_{4}$ requires 367.1406.

3-(4-amino-2-oxopyrimidin-1(2H)-yl)-N'-(3-methyl-4-oxonaphthalen-1(4H)-ylidene)propanehydrazide $(6 \mathrm{l})$ : Yellow solid, $70 \%$ yield, m.p. $>260^{\circ} \mathrm{C} .{ }^{1} \mathrm{H}$ NMR $(500 \mathrm{MHz}$, DMSO$\left.d_{6}\right): \delta 2.10\left(\mathrm{~s}, 3 \mathrm{H}, \mathrm{CH}_{3}-\mathrm{b}\right) ; 3.20\left(\mathrm{~m}, 2 \mathrm{H}, \mathrm{CH}_{2}-5\right) ; 3.97\left(\mathrm{~m}, 2 \mathrm{H}, \mathrm{CH}_{2}-4\right) ; 5.63(\mathrm{~d}, J=7.0 \mathrm{~Hz}$, $\left.1 \mathrm{H}, \mathrm{H}_{\mathrm{Ar}}\right) ; 7.02\left(\mathrm{~m}, 2 \mathrm{H}, \mathrm{NH}_{2}-3\right) ; 7.60-8.24\left(\mathrm{~m}, 6 \mathrm{H}, \mathrm{H}_{\mathrm{Ar}}\right) ; 11.66,11.91(\mathrm{~s}, 1 \mathrm{H}, \mathrm{NH}-6) .{ }^{13} \mathrm{C} \mathrm{NMR}$ $\left(500 \mathrm{MHz}, \mathrm{DMSO}-d_{6}\right): \delta 17.0\left(\mathrm{CH}_{3}-\mathrm{b}\right) ; 32.3\left(\mathrm{CH}_{2}-5\right) ; 45.6\left(\mathrm{CH}_{2}-4\right) ; 95.6\left(\mathrm{CH}_{\mathrm{Ar}}-1\right) ; 123.6$ $(\mathrm{C}=\mathrm{N}) ; 123.8\left(\mathrm{C}_{\mathrm{Ar}}\right) ; 124.5\left(\mathrm{CH}_{\mathrm{Ar}}\right) ; 126.6\left(\mathrm{CH}_{\mathrm{Ar}}-\mathrm{a}\right) ; 129.8\left(\mathrm{CH}_{\mathrm{Ar}}\right) ; 130.3\left(\mathrm{C}_{\mathrm{Ar}}\right) ; 133.2\left(\mathrm{CH}_{\mathrm{Ar}}\right)$; $134.3,136.5,139.3\left(\left[\mathrm{C}_{\mathrm{Ar}}\right]_{3}\right) ; 147.3\left(\mathrm{CH}_{\mathrm{Ar}}-2\right) ; 156.1\left(\mathrm{C}=\mathrm{O}_{\text {ketone }}\right) ; 166.3\left(\mathrm{C}=\mathrm{O}_{\text {amide }}\right) ; 184.7$ $\left([\mathrm{C}=\mathrm{O}]_{\text {ketone }}\right)$. FTIR $\left(\mathrm{cm}^{-1}\right): 796 \mathrm{~m}, 1220 \mathrm{~m}, 1280 \mathrm{w}, 1300 \mathrm{~m}, 1495 \mathrm{~s}, 1610 \mathrm{~s}, 1663 \mathrm{~s}, 3100 \mathrm{w}$. HRMS (ESI $\left.{ }^{+}, \mathrm{MeOH}\right)$ : Found: $m / z 352.1410\left[(\mathrm{M}+\mathrm{H})^{+}\right], \mathrm{C}_{18} \mathrm{H}_{18} \mathrm{~N}_{5} \mathrm{O}_{3}$ requires 352.1409. 
3-(6-amino-9H-purin-9-yl)-N'-(3-methyl-4-oxonaphthalen-1(4H)-ylidene)propanehydrazide $(6 m)$ :

Yellow solid, $65 \%$ yield, m.p. $253-255^{\circ} \mathrm{C} .{ }^{1} \mathrm{H}$ NMR $\left(500 \mathrm{MHz}, \mathrm{DMSO}-d_{6}\right): \delta 2.08(\mathrm{~s}, 3 \mathrm{H}$, $\mathrm{CH}_{3}$-b); 3.43 (m, 2H, $\mathrm{CH}_{2}$ ); 4.35, 4.51 (t, $J=6.5 \mathrm{~Hz}, 2 \mathrm{H}, \mathrm{CH}_{2}$ ); 7.19 (bs, $2 \mathrm{H}, \mathrm{NH}_{2}-3$ ); 7.61 $8.23\left(\mathrm{~m}, 7 \mathrm{H}, \mathrm{H}_{\mathrm{Ar}}\right) ; 11.63,11.95(\mathrm{~s}, 1 \mathrm{H}, \mathrm{NH}-6) .{ }^{13} \mathrm{C} \mathrm{NMR}\left(500 \mathrm{MHz}, \mathrm{DMSO}-d_{6}\right): \delta 16.5\left(\mathrm{CH}_{3}\right)$; $32.5\left(\mathrm{CH}_{2}\right) ; 66.2\left(\mathrm{CH}_{2}\right) ; 118.6,123.5,124.9,128.7,132.0,140.4(\mathrm{C}=\mathrm{N}) ; 151.7,155.3$ $\left([\mathrm{C}=\mathrm{O}]_{2}\right)$. FTIR $\left(\mathrm{cm}^{-1}\right): 642 \mathrm{~s}, 1168 \mathrm{~s}, 1253 \mathrm{~m}, 1285 \mathrm{~s}, 1394 \mathrm{~m}, 1650 \mathrm{~s}, 3171 \mathrm{~s} . \mathrm{HRMS}\left(\mathrm{ESI}^{+}\right.$, $\mathrm{MeOH}$ ): Found: $m / z 376.1522\left[(\mathrm{M}+\mathrm{H})^{+}\right], \mathrm{C}_{19} \mathrm{H}_{18} \mathrm{~N}_{7} \mathrm{O}_{2}$ requires 376.1523. LCMS (ESI ${ }^{+}, \mathrm{C} 18$ column, $\left.\mathrm{MeOH} 75 \% / \mathrm{H}_{2} \mathrm{O} 25 \%\right): m / z$ calcd for $\mathrm{C}_{19} \mathrm{H}_{16} \mathrm{~N}_{7} \mathrm{O}_{2}$ [(M-H) $\left.{ }^{-}\right]$374.1, found 374.0. HRMS (ESI $\left.{ }^{+}, \mathrm{MeOH}\right)$ : Found: $m / z 376.1523\left[(\mathrm{M}+\mathrm{H})^{+}\right], \mathrm{C}_{19} \mathrm{H}_{18} \mathrm{~N}_{7} \mathrm{O}_{2}$ requires 376.1522.

\subsection{Biologigal Assay}

The biological assay was conducted according to the procedure described in the literature. ${ }^{34}$ and is presented below.

\subsubsection{Cell Culture}

HCT116 p53+/+ human colon cancer cells and MCF-7 (p53+/+, noninvasive) human breast cancer cell lines were cultured in RPMI 1640 (Sigma-Aldrich, UK) with $20 \mathrm{mM}$ HEPES and L-Glutamine at $37^{\circ} \mathrm{C}$ in a humidified atmosphere of $5 \% \mathrm{CO}_{2}$ and $95 \%$ air. Media was supplemented with $1 \%$ Penicillin-Streptomycin $(100 \mathrm{U} / \mathrm{ml})$ and $10 \%$ heat-inactivated FBS (Sigma-Aldrich, Germany), $1 \%$ non essential amino acids and $1 \%$ of Na pyruvate.

\subsubsection{Cell Viability Assays}

The cells were seeded at $12 \times 10^{3}$ cells/well in 96-well plates and treated with different concentrations of compounds $\mathbf{6 a - j}$ for $24 \mathrm{~h}$. Control cells were treated with 6a-m such that the concentration of DMSO was less than $0.3 \%$. The inhibition of cell viability (expressed as percentage of control) was measured by the Cell Titer 96 non-radioactive cell proliferation kit (Promega Corp, Madison, Wisconsin, USA). This assay is an MTT based method that measures the ability of metabolically active cells to convert tetrazolium salt into a blue formazan product, and its absorbance is recorded at $595 \mathrm{~nm}$. Each value is the mean $\pm \mathrm{SD}$ of two separate experiments each done in triplicates. The $\mathrm{IC}_{50}$ represents the concentration at which $50 \%$ of the cells are viable.

\section{Results and Discussion}

\subsection{Chemistry}

Menadione, 2-methyl-1,4-naphthoquinone 4, undergoes reaction with acylhydrazides 5a-m via a nucleophilic addition reaction. Preparation of the desired menadione acylhydrazone derivatives 6a-m was achieved by a condensation reaction between menadione 4 and several aliphatic, aromatic and pyrimidine and purine nucleobase hydrazides. The reactant acylhydrazides were synthesized from the esters $7 \mathbf{a}-\mathbf{m}$ by an established literature procedure that involves the reaction of hydrazine hydrate in methanol with a catalytic amount of trifluoroacetic acid. ${ }^{35-37}$ The resultant hydrazides were then reacted with menadione in ethanol with a few drops of trifuluoroacetic acid at reflux for 24 (or 72) hours (Figure 2) using the conventional condensation method. ${ }^{38,39}$ The product hydrazones $\mathbf{6 a - m}$ were isolated as pure crystalline solids in good to excellent yields. 
$\overbrace{\mathbf{7 a - m}}^{\mathrm{O}} \overbrace{\mathrm{OCH}_{2} \mathrm{CH}_{3}}$

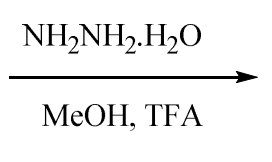

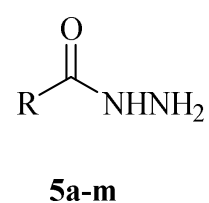

5a-m
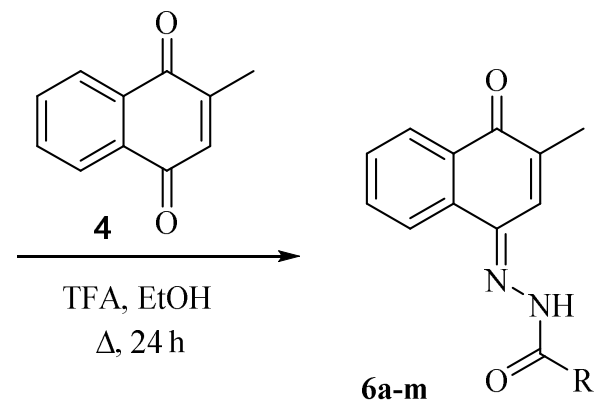

Figure 2. Scheme showing synthesis of acylhydrazones of menadione

Table 1. Synthesis of menadione acylhydrazone derivatives

\begin{tabular}{|c|c|c|c|c|c|}
\hline Product & $\begin{array}{c}\text { Hydrazide } \\
\left(\mathrm{RCONHNH}_{2}\right)\end{array}$ & $\begin{array}{c}\text { Reaction } \\
\text { Time }\end{array}$ & Yield & m.p. $\left({ }^{\circ} \mathrm{C}\right)$ & $\begin{array}{l}\text { Molecular } \\
\text { Formula }\end{array}$ \\
\hline $6 \mathbf{a}$ & NHI & $24 \mathrm{~h}$ & $76 \%$ & $243-245$ & $\mathrm{C}_{16} \mathrm{H}_{18} \mathrm{~N}_{2} \mathrm{O}_{2}$ \\
\hline $6 b$ & $\mathrm{HNH}$ & $24 \mathrm{~h}$ & $65 \%$ & 209-212 & $\mathrm{C}_{19} \mathrm{H}_{24} \mathrm{~N}_{2} \mathrm{O}_{2}$ \\
\hline $6 c$ & NHI & $24 \mathrm{~h}$ & $67 \%$ & $187-190$ & $\mathrm{C}_{22} \mathrm{H}_{28} \mathrm{~N}_{2} \mathrm{O}_{2}$ \\
\hline $6 d$ & IN & $24 \mathrm{~h}$ & $64 \%$ & 189-191 & $\mathrm{C}_{22} \mathrm{H}_{32} \mathrm{~N}_{2} \mathrm{O}_{2}$ \\
\hline $6 e$ & $\mathrm{NHNH}$ & $24 \mathrm{~h}$ & $60 \%$ & $180-182$ & $\mathrm{C}_{25} \mathrm{H}_{36} \mathrm{~N}_{2} \mathrm{O}_{2}$ \\
\hline $6 f$ & & $24 \mathrm{~h}$ & $79 \%$ & $166-169$ & $\mathrm{C}_{29} \mathrm{H}_{44} \mathrm{~N}_{2} \mathrm{O}_{2}$ \\
\hline
\end{tabular}


Table 1 Continued...

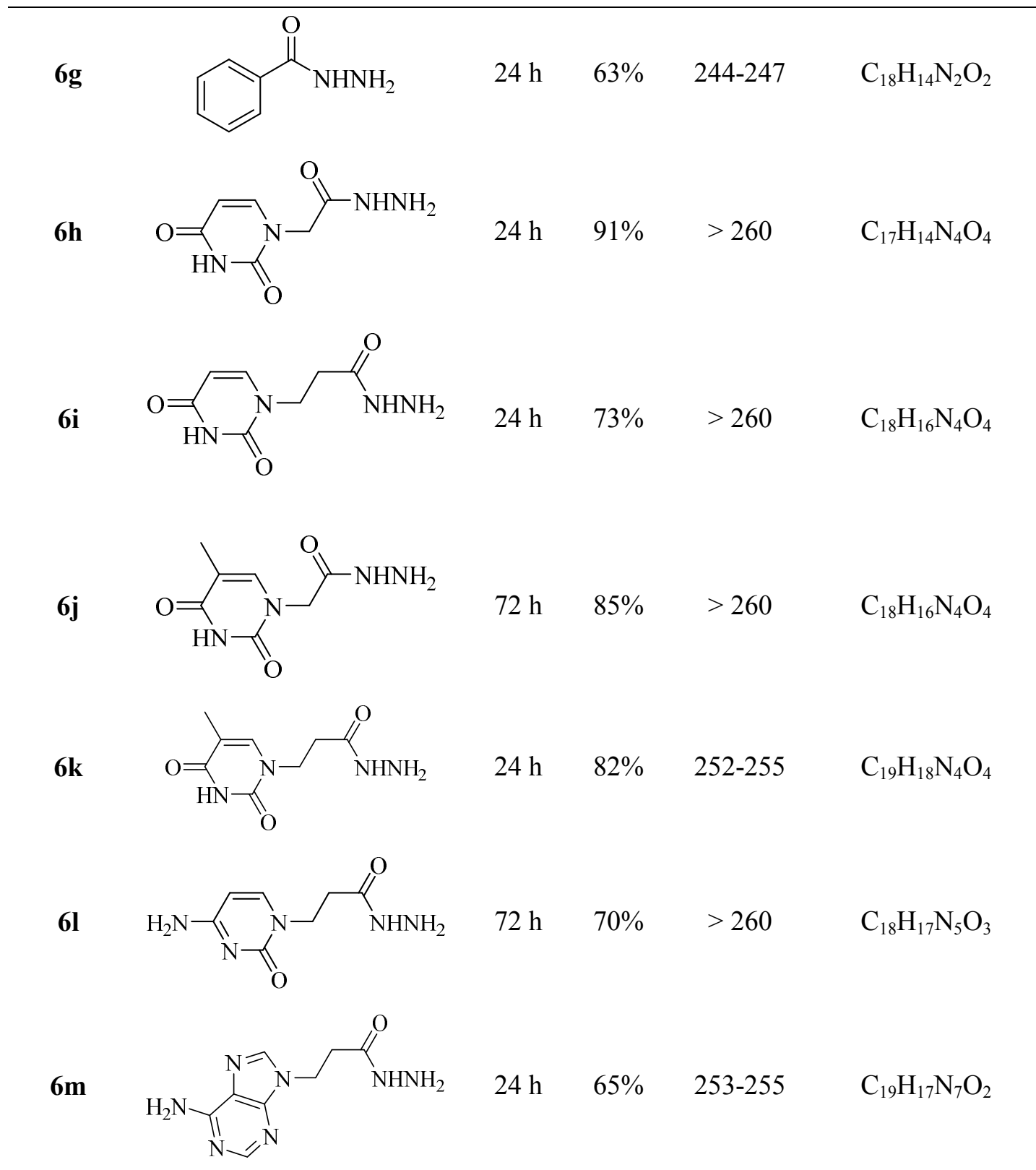

The novel menadione hydrazones 6a-m were identified and fully characterized by IR, ${ }^{1} \mathrm{H}$ NMR, ${ }^{13} \mathrm{C}$ NMR, 2D NMR spectroscopy and HRMS. The formation of the acylhydrazone functional group was supported by characteristic IR stretching frequencies in the range of $1680-1699$ and $1645-1650 \mathrm{~cm}^{-1}$, which represent $\mathrm{C}=\mathrm{O}$ and $\mathrm{C}=\mathrm{N}$ groups respectively. ${ }^{40}$

An interesting common detail of the NMR spectra of the acylhydrazones 6a-m is the appearance of a single set of proton and carbon resonance signals, despite the possible formation of regioisomers and the usual existence of isomers about the imine-like $\mathrm{C}=\mathrm{N}$ bond. The fact that hydrazones can exist as geometric $E / Z$-isomers about the $\mathrm{C}=\mathrm{N}$ double bond and as cis/trans-amide conformers means that overall, the acylhydrazone derivatives $\mathbf{6 a - m}$ can exist in four possible forms: two rotameric forms for each geometrical isomer as shown in Figure $3 .^{4-45}$ The presence of a single set of NMR signals in $\mathrm{d}_{6}$-DMSO indicates that only one geometric isomer and rotameric form exists in solution. 
<smiles>CC1=C/C(=N\NC(=O)c2ccccc2)c2ccccc2C1=O</smiles>

E-trans<smiles>CC1=CC(=NNC(=O)c2ccccc2)c2ccccc2C1=O</smiles>

E-cis<smiles>CC1=C/C(=N/NC(=O)c2ccccc2)c2ccccc2C1=O</smiles>

Z-trans<smiles>CC1=C/C(=N/NC(=O)c2ccccc2)c2ccccc2C1=O</smiles>

Z-cis

Figure 3. The possible isomeric and conformational structures of the product hydrazones

Structural elucidation was supported by a 2D NMR-NOESY spectrum of several menadione hydrazone products. Here the spectrum for the benzoic derivative $\mathbf{6 g}$ shows a spatial interaction between the hydrazone $\mathrm{NH}$ and the vinylic hydrogen of the naphthoquinone moiety (Figure 4). Expectedly, a spatial interaction between the NH and the methyl hydrogens was not observed.

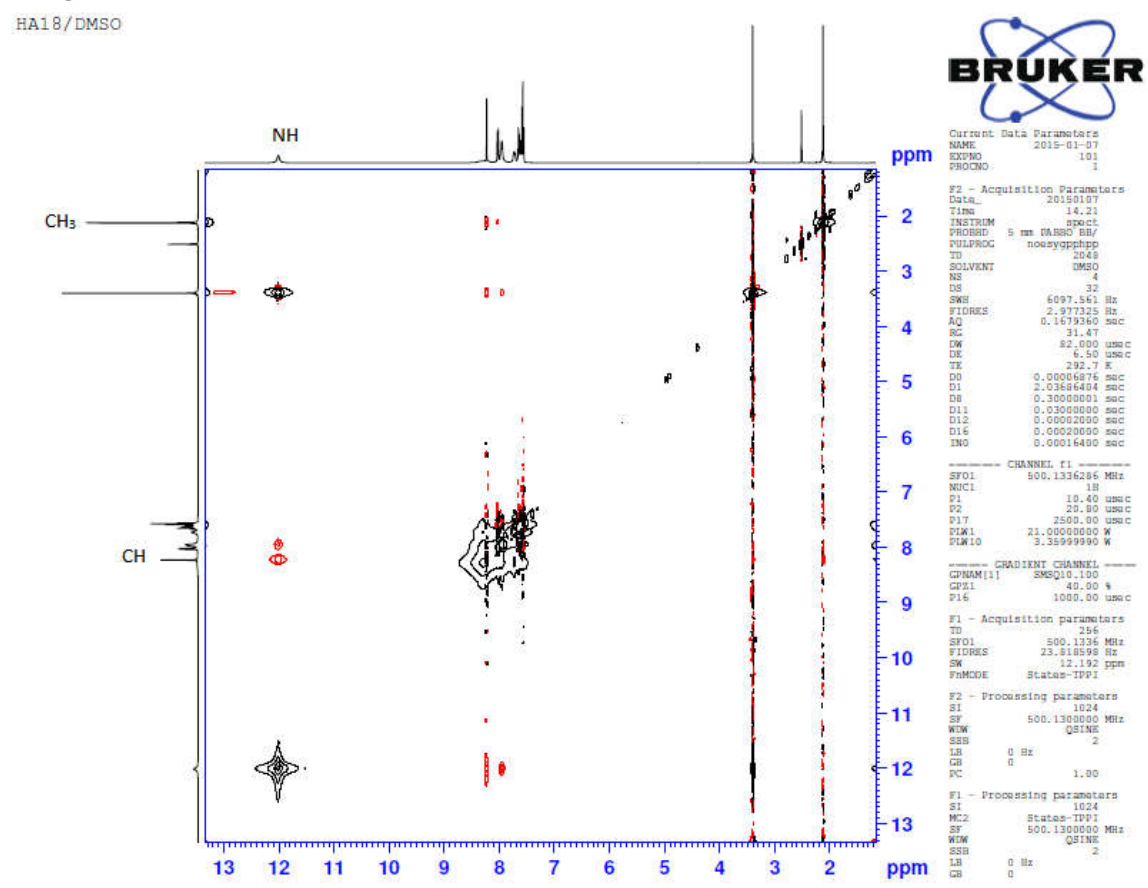

Figure 4. NOESY spectrum of $\mathbf{6 g}$

We postulated that the hydrazide coupled to the less hindered carbonyl carbon and has a conformation similar to 1a. This is consistent with the electronic effect whereby protonation of the menadione from the less hindered carbonyl carbon leads to a more stable tertiary allylic carbocation. However, protonation from the more hindered side leads to a less stable secondary allylic carbocation (Figure 5). 
<smiles>CC1=CC(=O)c2ccccc2C(=O)C1=O</smiles>

Figure 5. Structures of protonated menadione

The NOESY spectrum, which showed a spatial interaction between the hydrazone $\mathrm{NH}$ and the vinylic hydrogen and aromatic hydrogen, supported formation of the $E$ geometrical isomer and trans-conformer. This is consistent with multiple literature reports which state that the $E$-isomer is the predominant form in solution due to hindered rotation about the imine bond. ${ }^{41-45}$

Table 2. $\mathrm{IC}_{50}$ Values $(\mu \mathrm{M})$ of acylhydrazones 6a-m

\begin{tabular}{ccc}
\hline Compounds & HCT116 & MCF-7 \\
\hline $\mathbf{6 a}$ & $300 \pm 2.5$ & $71 \pm 2.5$ \\
$\mathbf{6 b}$ & $119 \pm 3.6$ & $269 \pm 9.4$ \\
$\mathbf{6 c}$ & $294 \pm 9.5$ & $90 \pm 2.6$ \\
$\mathbf{6 d}$ & $75 \pm 6.4$ & $56 \pm 6.4$ \\
$\mathbf{6 e}$ & $89 \pm 3.1$ & $100 \pm 1.2$ \\
$\mathbf{6 f}$ & $64 \pm 8.1$ & $62 \pm 3.4$ \\
$\mathbf{6 g}$ & $179 \pm 5.6$ & $56 \pm 2.6$ \\
$\mathbf{6 h}$ & $438 \pm 5.6$ & $250 \pm 4.8$ \\
$\mathbf{6 i}$ & $179 \pm 4.5$ & $100 \pm 4.2$ \\
$\mathbf{6 j}$ & $186 \pm 4.9$ & $265 \pm 5.9$ \\
$\mathbf{6 k}$ & $478 \pm 1.6$ & $225 \pm 9.4$ \\
$\mathbf{6}$ & $100 \pm 8.4$ & $73 \pm 1.8$ \\
$\mathbf{6 m}$ & $208 \pm 7.2$ & $104 \pm 3.2$ \\
\hline
\end{tabular}

\subsection{Biological Assay}

The cytotoxic activities of naphthoquinone and its derivatives are well documented in the literature. ${ }^{6-12}$ Thus, the naphthoquinone acylhydrazones $\mathbf{6 a - m}$ were assessed for their anticancer effects against MCF-7 and HCT116 cell lines using MTT viability assay. The results of the compounds after $24 \mathrm{~h}$ of treatment are summarized in Figure 6. Most compounds showed dose-dependent inhibitory effects on the two cancer cell lines and were active at concentrations higher than $100 \mu \mathrm{M}$. The most effective compounds on HCT116 was 6e and $\mathbf{6 f}$, with $\mathrm{IC}_{50}$ of 89 and $64 \mu \mathrm{M}$. Interestingly, these two acylhydrazones, $6 \mathbf{e}$ and $\mathbf{6 f}$, contain the longest hydrocarbon chains which may have contributed to increased cell permeability and therefore elevated biological activity compared to the other hydrazones. On the other hand, the most biologically active molecules on MCF-7 were $\mathbf{6 a}, \mathbf{6 c}, \mathbf{6 d}, \mathbf{6 f}, \mathbf{6 g}$ and $6 \mathbf{1}$ with $\mathrm{IC}_{50}$ of $70,90,56,62,56$ and $73 \mu \mathrm{M}$, respectively (Table 2). There appears to be no 
apparent connection between potency and structural features though the most active molecules on MCF-7 cells are lauric-menadione 6d, benzoic hydrazone $\mathbf{6 g}$. Notably, the stearic hydrazone 6 also greatly decreased viability in MCF-7 cell lines $\left(\mathrm{IC}_{50} 62 \mu \mathrm{M}\right)$ which may be attributed to its lipophilicity. In general, the activity of the compounds varied depending on cell type indicating cell type specificity, implying that the molecules interact differently with the different cancer cells.
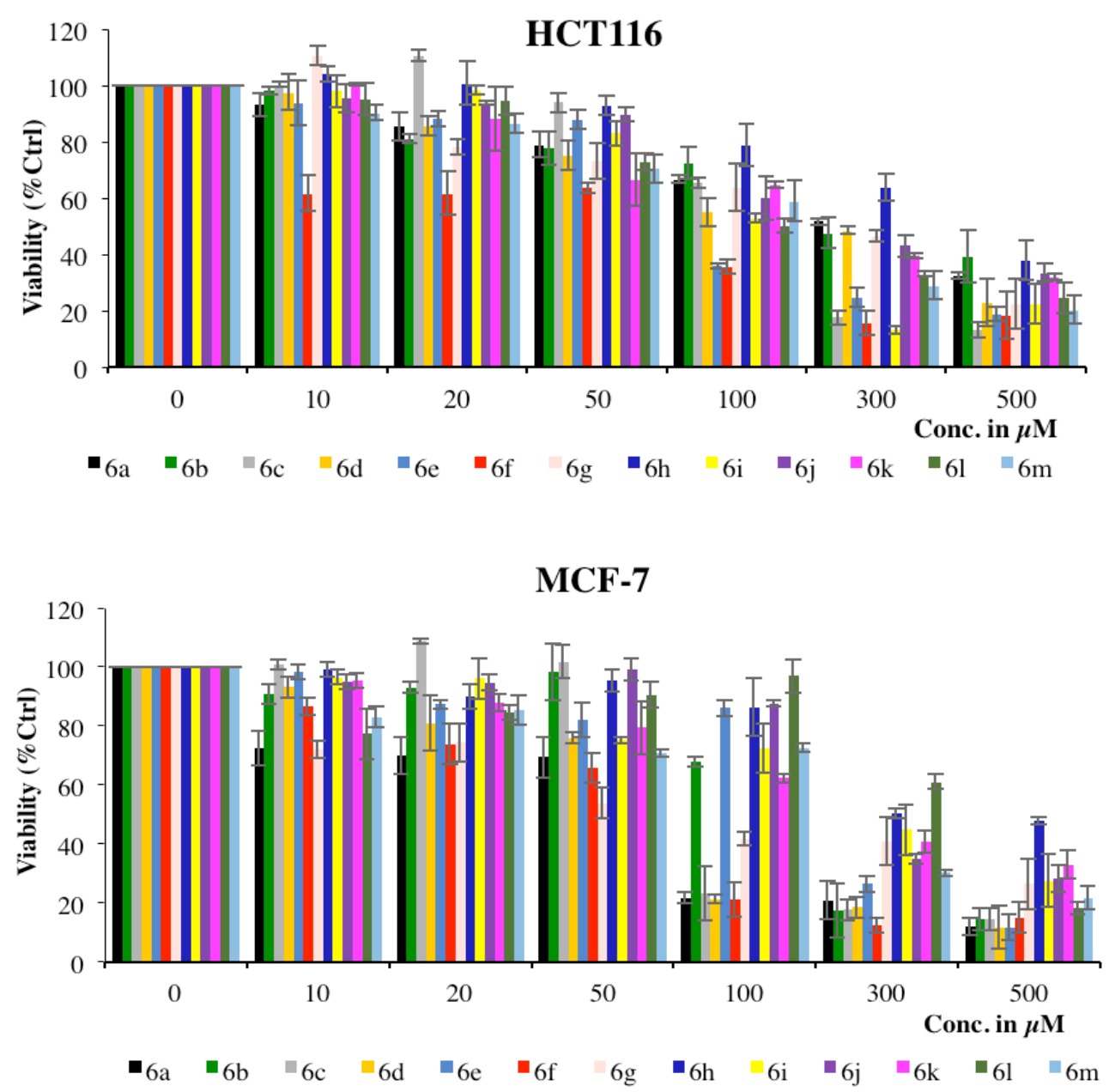

Figure 6. Cell viability of HCT116 and MCF-7 cells upon treatment with 6a-m as determined by MTT assay.

\section{Conclusion}

The simple and direct preparation of a novel library of 2-methyl-1,4-naphthoquinone acylhydrazone derivatives is described. The hydrazone products were synthesized in good to excellent yields by an acid-catalyzed condensation of numerous alkyl and aromatic hydrazides with menadione in refluxing ethanol. Various spectroscopic methods confirmed formation of the product hydrazones and these included ${ }^{1} \mathrm{H} N M R,{ }^{13} \mathrm{C}$ NMR, 2D NMR IR, LC-MS and HRMS. Biological assays indicated that the menadione acylhydrazones exhibited significant anticancer activity, since colon and breast cancer cell viability was greatly reduced after treatment with these novel molecules. Specifically, the hydrazones containing highly 
lipophilic hydrocarbon chains - those derived from myristic and stearic hydrazide - proved to be the most cytotoxic against HCT116 cancer cells.

\section{Acknowledgements}

This study was financially supported by the Lebanese National Council for Scientific Research (LNCSR) and the Lebanese American University School Research and Development Council (LAU SRDC).

\section{Supporting Information}

Supporting Information accompanies this paper on http://www.acgpubs.org/OC

\section{ORCID}

Kamal Bou Hadir: 0000-0002-6375-1763

Hala Atallah: 0000-0001-8349-4402

Rana Mezher: 0000-0002-5488-5356

Maamoun Fatfat: 0000-0001-9642-6708

Hala Gali-Muhtasib: 0000-0001-6840-3015

Jomana Elaridi: 0000-0002-9198-8952

\section{References}

[1] Pinto, A. V.; De Castro, S. L. The trypanocidal activity of naphthoquinones: A review. Molecules 2009, 14(11), 4570-4590.

[2] Tandon, V. K.; Maurya, H. K.; Mishra, N. N.; Shukla, P. K. Design, synthesis and biological evaluation of novel nitrogen and sulfur containing hetero-1,4-naphthoquinones as potent antifungal and antibacterial agents. Eur. J. Med. Chem. 2009, 44(8), 3130-3137.

[3] Huang, S-T.; Kuo, H-S.; Hsiao, C-L.; Lin, Y-L. Efficient synthesis of "redox-switched" naphthoquinone thiol-crown ethers and their biological activity evaluation. Bioorg. Med. Chem. 2002, 10(6), 1947-1952.

[4] da Silva, A. J.; Buarque, C. D.; Brito, F. V.; Aurelian, L.; Macedo, L. F.; Malkas, L. H.; Hickey, R. J.; Lopes, D. V.; Noël, F.; Murakami, Y. L. Synthesis and preliminary pharmacological evaluation of new $( \pm$ ) 1,4-naphthoquinones structurally related to lapachol. Bioorg. Med. Chem. 2002, 10(8), 2731-2738.

[5] García-Barrantes, P. M.; Lamoureux, G. V.; Pérez, A. L.; García-Sánchez, R. N.; Martínez, A. R.; San Feliciano, A. Synthesis and biological evaluation of novel ferrocene-naphthoquinones as antiplasmodial agents. Eur. J. Med. Chem. 2013, 70, 548-557.

[6] Seshadri, P.; Rajaram, A.; Rajaram, R. Plumbagin and juglone induce caspase-3-dependent apoptosis involving the mitochondria through ROS generation in human peripheral blood lymphocytes. Free Radic. Biol. Med. 2011, 51(11), 2090-2107.

[7] Bhasin, D; Chettiar, S. N.; Etter, J. P.; Mok, M.; Li, P. K. Anticancer activity and SAR studies of substituted 1,4-naphthoquinones. Bioorg. Med. Chem.. 2013, 21(15), 4662-4669.

[8] Pérez-Sacau, E.; Díaz-Peñate, R. G.; Estévez-Braun, A.; Ravelo, A. G.; García-Castellano, J. M.; Pardo, L.; Campillo, M. Synthesis and pharmacophore modeling of naphthoquinone derivatives with cytotoxic activity in human promyelocytic leukemia HL-60 cell Line. J. Med. Chem. 2007, 50(4), 696-706.

[9] Jiménez-Alonso, S.; Orellana, H. C.; Estévez-Braun, A.; Ravelo, A. G.; Pérez-Sacau, E.; Machín, F. Design and synthesis of a novel series of pyranonaphthoquinones as topoisomerase II catalytic inhibitors. J. Med. Chem. 2008, 51(21), 6761-6772.

[10] Klaus, V.; Hartmann, T.; Gambini, J.; Graf, P.; Stahl, W.; Hartwig, A.; Klotz, L. O. 1,4Naphthoquinones as inducers of oxidative damage and stress signaling in HaCaT human 
keratinocytes. Arch. Biochem. Biophys. 2010, 496(2), 93-100.

[11] Bustamante, F. L. S.; Metello, J. M.; de Castro, F. A. V.; Pinheiro, C.B.; Pereira, M. D.; Lanznaster, M. Lawsone Dimerization in cobalt(III) complexes toward the design of new prototypes of bioreductive prodrugs. Inorg. Chem. 2013, 52(3), 1167-1169.

[12] Oramas-Royo, S.; Torrejón, C.; Cuadrado, I.; Hernández-Molina, R.; Hortelano, S.; EstévezBraun, A.; De Las Heras, B. Synthesis and cytotoxic activity of metallic complexes of lawsone. Biorg. Med. Chem. 2013, 21(9), 2471-2477.

[13] Checker, R.; Sharma, D.; Sandur, S. K.; Khanam, S.; Poduval, T. B. Anti-inflammatory effects of plumbagin are mediated by inhibition of NF-kappaB activation in lymphocytes. Int. Immunopharmacol. 2009, 9(7-8), 949-958.

[14] López-López, L.; Nery-Flores, D.; Silva-Belmares, Y.; Sáenz-Galindo, A. Naphthoquinones: Biological properties and synthesis of lawsone and derivatives - A structured review. Vitae Rev. La Fac. Quim. Farm. 2014, 21(13), 248-258.

[15] Daines, A. M.; Payne, R. J.; Humphries, M. E.; Abell, A. D. The synthesis of naturally occurring vitamin K and vitamin K analogues. Curr. Org. Chem. 2003, 7(16), 1625-1634.

[16] Salomi, M. J.; Nair, S. C.; Panikkar, K. R. Inhibitory effects of Nigella sativa and saffron (crocus sativus) on chemical carcinogenesis in mice. Nutr. Cancer 1991, 16(1), 67-72.

[17] Salomi, N. J.; Nair, S. C.; Jayawardhanan, K. K.; Varghese, C. D.; Panikkar, K. R. Antitumor principles from Nigella sativa seeds. Cancer Lett. 1992, 63(1), 41-46.

[18] Shoieb, A. M.; Elgayyar, M.; Dudrick, P. S.; Bell, J. L.; Tithof, P. K. In vitro inhibition of growth and induction of apoptosis in cancer cell lines by thymoquinone. Int. J. Oncol. 2003, 22(1), 107113.

[19] Gali-Muhtasib, H.; Diab-Assaf, M.; Boltze, C.; Al-Hmaira, J.; Hartig, R.; Roessner, A.; SchneiderStock, R. Thymoquinone extracted from black seed triggers apoptotic cell death in human colorectal cancer cells via a p53-dependent mechanism. Int. J. Oncol. 2004, 25(4), 857-866.

[20] Gali-Muhtasib, H.; Aboukheir, W.; Kheir, L.; Darwiche, N.; Crooks, P. Molecular pathway for thymoquinone-induced cell cycle arrest and apoptosis in neoplastic keratinocytes. Anticancer Drugs 2004, 15, 389-399.

[21] Gali-Muhtasib, H.; El-Najjar, N.; Schneider-Stock, R. The medicinal potential of black seed (Nigella sativa) and its components. Adv. Phytomed. 2006, 133-153.

[22] Schobert, R.; Breyer, S.; Effenberger, K. Effects of thymoquinone - Fatty acid conjugates on cancer cells. Chem.Med.Chem. 2009, 4(5), 761-768.

[23] Rollas, S.; Küçükgüzel, Ş. G. Biological activities of hydrazone derivatives. Molecules 2007, 1910-1939.

[24] Chimenti, F.; Maccioni, E.; Secci, D.; Bolasco, A.; Chimenti, P.; Granese, A.; Befani, O.; Turini, P.; Alcaro, S.; Ortuso, F.; Cardia, M. C.; Distinto, S. Selective inhibitory activity against MAO and molecular modeling studies of 2-thiazolylhydrazone derivatives. J. Med. Chem. 2007, 50(4), 707-712.

[25] Mao, J.; Wan Baojie, Y. W.; Kozikowski, A. P.; Franzblau, S. G. Design, synthesis, and pharmacological evaluation of mefloquine-based ligands as novel antituberculosis agents. ChemMedChem 2007, 2(11), 1624-1630.

[26] Noulsri, E.; Richardson, D. R.; Lerdwana, S.; Fucharoen, S.; Yamagishi, T.; Kalinowski, D. S.; Pattanapanyasat, K. Antitumor activity and mechanism of action of the iron chelator, Dp44mT, against leukemic cells. Am. J. Hematol. 2009, 84, 170-176.

[27] Vicini, P.; Incerti, M.; Doytchinova, I. A.; La Colla, P.; Busonera, B.; Loddo, R. Synthesis and antiproliferative activity of benzo[d]isothiazole hydrazones. Eur. J. Med. Chem. 2006, 41(5), 624-632.

[28] Andreani, A.; Burnelli, S.; Granaiola, M.; Leoni, A.; Locatelli, A.; Morigi, R.; Rambaldi, M.; Varoli, L.; Calonghi, N.; Cappadone, C.; Farruggia, G.; Zini, M.; Stefanelli, C.; Masotti, L.; Radin, N. S.; Shoemaker, R. H. New antitumor imidazo[2,1-b]thiazole guanylhydrazones and analogues. J. Med. Chem. 2008, 51(4), 809-816.

[29] Najjar, V.A., editor. Developments in molecular and cellular biochemistry. In: The biological effects of glutamic acid and its derivatives. Netherlands: Springer; 1981. p. 99.

[30] Miller, A.; Connor, B. The carboxylation activity of vitamin with substitutions. J. Biol. Chem. 1979, 254(8), 2656-2664.

[31] Bourderioux, A.; Nauš, P.; Perlíková, P.; Pohl, R.; Pichová, I.; Votruba, I.; Džubák, P.; Konečný, P.; Hajdúch, M.; Stray, K. M.; Wang, T.; Ray, A. S.; Feng, J. Y.; Birkus, G.; Cihlar, T.; Hocek, M. Synthesis and significant cytostatic activity of 7-hetaryl-7-deazaadenosines. J. Med. Chem. 
2011, 54 (15), 5498-5507.

[32] Galmarini, C. M.; Mackey, J. R.; Dumontet, C. Nucleoside analogues and nucleobases in cancer treatment. Lancet Oncol. 2002, 415-424.

[33] Parker, W. B.; Secrist, J. A.; Waud, W. R. Purine nucleoside antimetabolites in development for the treatment of cancer. Curr. Opin. Investig. Drugs 2004, 5 (6), 592-596.

[34] a) Elaridi, J.; Karroum, N. B.; Bouhadir, K.; Fatfat, M.; Gali-Muhtasib, H. Preparation and biological evaluation of novel acylhydrazide derivatives of 2,3-dichloronaphthoquinone. Org. Commun. 2017, 10 (2),122-129.; b) Aminudin N.I.; Ahmad, F.; Taher, M.; Zulkifli, R.M. Cytotoxic and antibacterial activities of constituents from Calophyllum ferrugineum Ridley. Rec. Nat. Prod. 2016,10,649-653.

[35] Rauf, A.; Sharma, S.; Gangal, S. Microwave assisted efficient one-pot synthesis of 3,5,6trisubstituted-1,2,4-triazines from fatty acid hydrazides under solvent-free conditions and their antimicrobial activity. ARKIVOC 2007, 16, 137-147.

[36] Markley, K. S. Fatty acids: Their chemistry, properties, production and uses, Part 3. Interscience Publishers, John Wiley and Sons: New York, 1964.

[37] Awasthi, S.; Rishishwar, P.; Rao, A. N.; Ganesan, K.; Malhotra, R. C. Synthesis, characterization and spectral studies of various newer long chain aliphatic Acid (2-hydroxy benzylidene and 1Hindol-3-ylmethylene) hydrazides as mosquito para-pheromones. J. Korean Chem. Soc. 2007, 51(6), 506-512.

[38] Ishida, T.; Shinozuka, K.; Nogami, T.; Kubota, M.; Ohashi, M. Synthesis and characterization of C60 derivatives possessing TEMPO radicals. Tetrahedron 1996, 52 (14), 5103-5112.

[39] Bouhadir, K. H.; Aleiwe, B. A.; Fares, F. A. Facile preparation of the tosylhydrazone derivatives of a series of racemic trans-3,4-substituted cyclopentanones. Molecules 2012, 17 (1), 1-14.

[40] Lin-Vien, D.; Colthup, N. B.; Fateley, W. G.; Grasselli, J. G. The handbook of infrared and raman characteristic frequencies of organic molecules. Academic Press, Elsevier Inc.: New York, 1991.

[41] Özdemir, A.; Turan-Zitouni, G.; Kaplancikli, Z. A.; Altintop, M. D. The synthesis of some new hydrazone derivatives containing the benzothiazole moiety. J. Serbian Chem. Soc. 2012, 77(2), 141-146.

[42] Palla, G.; Predieri, G.; Domiano, P.; Vignali, C.; Turner, W. Conformational behaviour and E/Z isomerization of $N$-acyl and $N$-aroylhydrazones. Tetrahedron 1986, 42(13), 3649-3654.

[43] Patorski, P.; Wyrzykiewicz, E.; Bartkowiak, G. Synthesis and conformational assignment of N(E)-stilbenyloxymethylenecarbonyl-substituted hydrazones of acetone and $o-(m-$ and $p-)$ chloro(nitro-)benzaldehydes by means of NMR spectroscopy. J. Spectrosc. 2013, 1-12.

[44] Nakka, M.; Begum, M. S.; Varaprasad, B. F. M.; Reddy, L. V.; Bhattacharya, A.; Helliwell, M.; Mukherjee, A. K.; Beevi, S. S.; Mangamoori, L. N.; Mukkanti, K.; Pal, S. Naproxen and ibuprofen based acyl hydrazone derivatives: Synthesis, structure analysis and cytotoxicity studies. J. Chem. Pharm. Res. 2010, 2(6), 393-409.

[45] Wyrzykiewicz, E.; Prukała, D. New isomeric N-substituted hydrazones of 2-, 3-and 4pyridinecarboxaldehydes. J. Heterocycl. Chem. 1998, 35(2), 381-387.

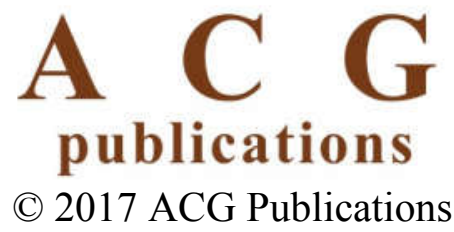

